\title{
Supercharging Protein Complexes from Aqueous Solution Disrupts their Native Conformations
}

\author{
Harry J. Sterling, ${ }^{1}$ Alexander F. Kintzer, ${ }^{1}$ Geoffrey K. Feld, ${ }^{1}$ Catherine A. Cassou, ${ }^{1}$ \\ Bryan A. Krantz, ${ }^{1,2}$ Evan R. Williams ${ }^{1}$ \\ ${ }^{1}$ Department of Chemistry, University of California-Berkeley, Berkeley, CA 94720, USA \\ ${ }^{2}$ Department of Molecular and Cell Biology, University of California-Berkeley, Berkeley, CA, USA
}

\begin{abstract}
The effects of aqueous solution supercharging on the solution- and gas-phase structures of two protein complexes were investigated using traveling-wave ion mobility-mass spectrometry (TWIMS-MS). Low initial concentrations of $m$-nitrobenzyl alcohol ( $m$-NBA) in the electrospray ionization (ESI) solution can effectively increase the charge of concanavalin A dimers and tetramers, but at higher $m$-NBA concentrations, the increases in charge are accompanied by solution-phase dissociation of the dimers and up to a $\sim 22 \%$ increase in the collision cross section (CCS) of the tetramers. With just $0.8 \% \mathrm{~m}$-NBA added to the ESI solution of a $\sim 630 \mathrm{kDa}$ anthrax toxin octamer complex, the average charge is increased by only 4\% compared with the "native" complex, but it is sufficiently destabilized so that extensive gas-phase fragmentation occurs in the relatively high pressure regions of the TWIMS device. Anthrax toxin complexes exist in either a prechannel or a transmembrane channel state. With $m$-NBA, the prechannel state of the complex has the same CCS/charge ratio in the gas phase as the transmembrane channel state of the same complex formed without $m$-NBA, yet undergoes extensive dissociation, indicating that destabilization from supercharging occurs in the ESI droplet prior to ion formation and is not a result of Coulombic destabilization in the gas phase as a result of higher charging. These results demonstrate that the supercharging of large protein complexes is the result of conformational changes induced by the reagents in the ESI droplets, where enrichment of the supercharging reagent during droplet evaporation occurs.
\end{abstract}

Key words: Electrospray ionization, Supercharging, Protein complex, Anthrax toxin, Concanavalin A, Traveling wave ion mobility, Native mass spectrometry, Charge enhancement

\section{Introduction}

$\mathrm{E}$ lectrospray ionization (ESI) mass spectrometry (MS) of

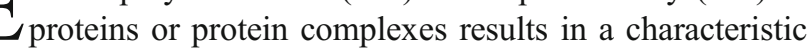

Electronic supplementary material The online version of this article (doi:10.1007/s13361-011-0301-y) contains supplementary material, which is available to authorized users.

Correspondence to: Evan R. Williams; e-mail: williams@cchem.berkeley. edu distribution of charge states from which information about the solution-phase conformations of the analytes can be inferred [1]. For aqueous or buffered aqueous solutions where the protein or complex has a "native" or "native-like" set of conformations, ESI typically results in narrow, lowcharge distributions, whereas the distributions from solutions that are more denaturing are typically broad and highcharge. In addition to the solution-phase conformations of the analyte $[2,3]$, many factors can affect the ion charge state distribution, including analyte and solvent basicities 
[4-6], solvent surface tension [7-9], and various instrument parameters [10-13]. Controlling these factors to reduce or increase ion charge can be of great analytical utility. For example, a number of methods have been shown to lower ion charge states $[5,6,14-16]$, facilitating identification of components from heterogeneous mixtures that can have complex mass spectra because of high peak densities.

Increasing ion charge can be highly desirable because the performance of most mass analyzers, and the efficiency of most tandem-MS techniques, improve with higher analyte charge. One method for increasing the charge states of peptides, proteins, and protein complexes is the addition of a small quantity of a low-volatility "supercharging" reagent to the ESI solution [7-9, 1734]. In both "denaturing" and "native" ESI solutions, supercharging reagents, such as $m$-nitrobenzyl alcohol ( $m$-NBA) [7], can cause significant charge enhancement compared with the same solutions without the reagent. During evaporation, ESI droplets become enriched with the reagent due to its very high boiling point relative to water and/or the organic solvents and acids that are typically used [35-37]. For example, the boiling point of $m$-NBA is $177{ }^{\circ} \mathrm{C}$ at 3 Torr [38], equal to $\sim 405{ }^{\circ} \mathrm{C}$ at 760 Torr [39]. In "denaturing" solutions containing acetic acid and relatively high concentrations of organic solvents, in which most proteins are more unfolded, these reagents increase the droplet surface tension as the more volatile components preferentially evaporate $[8,9]$. This enrichment results in an ESI droplet with a higher surface tension and thus a higher charge density needed to reach the Rayleigh limit [40]. More highly charged ions can be formed from droplets with a higher charge density. In contrast, the enrichment of the supercharging reagent lowers the surface tension of droplets in purely aqueous or buffered aqueous solutions (surface tension of $m$-NBA is $50 \pm 5 \mathrm{mN} / \mathrm{m}$ [8] compared with $72 \mathrm{mN} / \mathrm{m}$ at $25{ }^{\circ} \mathrm{C}$ for water [38]). In the absence of other factors that affect charging, this should cause a decrease in the analyte charge, as occurs for an amine-functionalized dendrimer, poly(propyleneimine) (DAB-16) [9], which cannot undergo significant conformational changes [41]. In contrast, when this molecule was electrosprayed from a methanol solution containing $m$-NBA (methanol surface

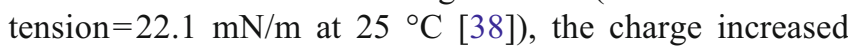
compared with the same solution without the reagent [9].

We have proposed that the increase in charge observed for proteins and protein complexes supercharged from purely aqueous or buffered aqueous solutions results primarily from chemical and/or thermal denaturation of the analyte that occurs in the ESI droplet during solvent evaporation, and that the charge enhancement from the conformational changes of the analyte can overcome the charge-reducing effects of lower droplet surface tension [1923]. Using circular dichroism spectroscopy (CD) to monitor the transition of equine myoglobin from folded to unfolded states, the denaturing strength of sulfolane, a compound found by Loo and coworkers to be an effective supercharging reagent [24], was measured to be $\sim 1.5 \mathrm{kcal} / \mathrm{mol} / \mathrm{M}$ at $25{ }^{\circ} \mathrm{C}$, which is $\sim 30 \%$ as effective as guanidinium chloride (a common denaturant in protein folding studies) for unfolding this protein [21]. In addition, the denaturing strength of sulfolane increases at higher temperatures [21]. Reagent enrichment increases the ESI droplet lifetime [35], which should result in an increased number of collisions with neutral gas molecules in the atmosphere/vacuum interface. This should, in turn, reduce the rate of evaporative cooling and increase the effective temperature of the droplet [19]. In addition to CD [21, 22], results from experiments using thermal activation [19], hydrogen/deuterium exchange-MS [22, 23], traveling wave ion mobility-MS (TWIMS) [22, 23], and chemical cross-linking [20] are consistent with the hypothesis that chemical and/or thermal denaturation of the analyte in the ESI droplet is the primary origin of aqueous solution supercharging of proteins and complexes.

Here, we use TWIMS-MS to examine the effects that low initial concentrations of $m$-NBA have on the solution- and gas-phase conformations of the dimers and tetramers of the $25 \mathrm{kDa}$ protein concanavalin $\mathrm{A}$ and an anthrax toxin octameric complex $(\sim 630 \mathrm{kDa})$. At low initial reagent concentrations, all of the complexes can be supercharged modestly, but at higher initial reagent concentrations, all of the complexes undergo disruptions of the native conformations that manifest as either solution- or gas-phase dissociation or as an increase in their collision cross sections.

\section{Experimental}

Mass spectra and TWIMS arrival time distributions were acquired using a hybrid quadrupole/ion mobility/time-offlight instrument (Synapt G2 High Definition Mass Spectrometer; Waters, Milford, MA, USA) equipped with a Zspray ion source. Ions were formed using nanoelectrospray emitters prepared by pulling borosilicate capillaries $(1.0 \mathrm{~mm}$ o.d./0.78 mm i.d.; Sutter Instruments, Novato, CA, USA) to a tip i.d. of $\sim 1 \mu \mathrm{m}$ with a Flaming/Brown micropipette puller (Model P-87; Sutter Instruments). A platinum wire (0.127 mm diameter; Sigma, St. Louis, MO, USA) was placed in the nanoelectrospray emitter in contact with the solution, and ESI was initiated and maintained by applying $\sim 1 \mathrm{kV}$ to the wire relative to instrument ground. Solutions of concanavalin A were prepared from lyophilized protein (Sigma; used without further purification) and solutions of anthrax toxin complexes were prepared as described in detail elsewhere [42]. $m$-NBA was from Sigma and was used without further purification.

TWIMS arrival times for all ions were assigned as the center of the full-width half-maximum (FWHM) of the arrival time distribution peak. Arrival times were used to calculate the collision cross sections utilizing the calibration protocol of Bush et al. [43]. Homotetramers of concanavalin A and alcohol dehydrogenase and 14-mers of GroEL formed 
from $\sim 10 \mu \mathrm{M}$ solutions were used as calibrant ions. Average charge was calculated as the abundance-weighted average of each charge state in a given charge state distribution. Relative abundances of concanavalin A monomers and oligomers were calculated from the sum of the peak areas for each species, and because of differences in ionization and detection efficiencies, are only useful for comparisons of changes in their relative abundances. For concanavalin A monomer and dimer peaks that have the same $\mathrm{m} / \mathrm{z}$, the relative contributions of each were obtained from the normalized intensities of the TWIMS arrival time distributions.

For the concanavalin A crosslinking experiments, lyophilized protein (Sigma) was dissolved in crosslinking buffer composed of $18 \mathrm{mM}$ potassium phosphate and $60 \mathrm{mM}$ sodium chloride, with the $\mathrm{pH}$ adjusted to 6.8 with sodium hydroxide. The final protein concentration was $90 \mu \mathrm{M}$. An identical solution was prepared but with the addition of $m$-NBA to $1 \%$ vol/vol. Suberic acid bis(3-sulfohydroxysuccinimide ester) (Sigma) dissolved in extra dry DMSO (Acros Organics) was added to both solutions at a final concentration of $480 \mu \mathrm{M}$ and allowed to react for $30 \mathrm{~min}$ at room temperature. The crosslinking reaction was quenched with the addition of ammonium acetate to a final concentration of $33 \mathrm{mM}$. The solutions were dialyzed against $20 \mathrm{mM}$ ammonium acetate $(\mathrm{pH}$ 7.0). After dialysis, aliquots were diluted 1:10 with neat formic acid and nanoelectrosprayed directly.

Collision cross section calculations of the anthrax toxin prechannel complexes were performed with the projection approximation algorithm in MOBCAL [44]. For the octamer prechannel complex, $\mathrm{PA}_{8}\left(\mathrm{LF}_{\mathrm{N}}\right)_{4}$, the crystal structure coordinates (PDB code $3 \mathrm{KWV}$; [45]) were used directly in the calculation. For the heptamer prechannel complex, $\mathrm{PA}_{7}\left(\mathrm{LF}_{\mathrm{N}}\right)_{3}$, the calculation was performed with coordinates generated from a homology model built using COOT [46] with the crystal structure coordinates of the PA heptameric ring (PDB code 1TZO; [47]) and three $\mathrm{LF}_{\mathrm{N}}$ molecules docked onto the ring by Least Squares Quotient alignment of $\mathrm{PA}_{2} \mathrm{LF}_{\mathrm{N}}$ ternary complexes from the $\mathrm{PA}_{8}\left(\mathrm{LF}_{\mathrm{N}}\right)_{4}$ coordinates (PDB code 3KWV; [45]). Prechannel and channel state models were made in CHIMERA [46] using PDB structures $3 \mathrm{KWV}$ and as a hybrid with $3 \mathrm{KWV}$ and $1 \mathrm{~V} 36$, respectively.

\section{Results and Discussion}

\section{Effects of Supercharging and Ionic Strength on Charge, Ion Abundance, and Collision Cross Section}

Concanavalin A from Canavalia ensiformis is a $25.6 \mathrm{kDa}$ protein that reversibly self-assembles to form dimers and tetramers in relative abundances that depend on solution $\mathrm{pH}$, temperature, and ionic strength [48-50]. Nanoelectrospray mass spectra of $\sim 10 \mu \mathrm{M}$ concanavalin $\mathrm{A}$ in $200 \mathrm{mM}$ ammonium acetate, $\mathrm{pH} 6.8$, solutions containing $0 \%-1.5 \%$ $m$-NBA are shown in Figure $1 \mathrm{a}-\mathrm{d}$. The mass spectrum
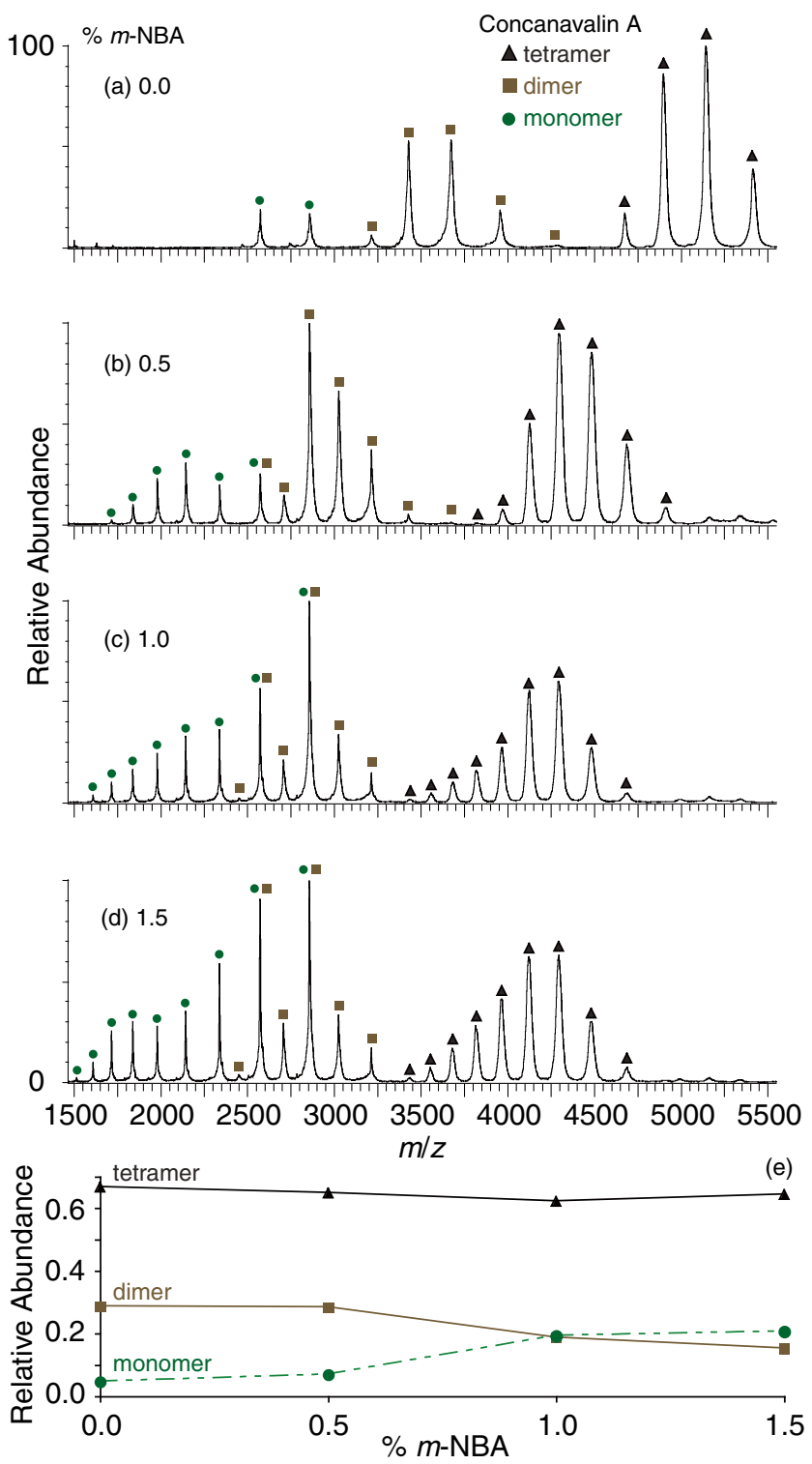

Figure 1. Nanoelectrospray mass spectra of $\sim 10 \mu \mathrm{M}$ concanavalin $\mathrm{A}$ in $200 \mathrm{mM}$ ammonium acetate, $\mathrm{pH}$ 6.8, aqueous solution containing (a) $0 \%$, (b) $0.5 \%$, (c) $1.0 \%$, and (d) $1.5 \% \mathrm{~m}$-NBA. The normalized abundances of concanavalin A monomer, dimer, and tetramer as a function of $m$-NBA concentration are shown in (e)

obtained from the electrospray solution without $m$-NBA (Figure 1a) is dominated by relatively narrow charge state distributions for tetramer (black triangles) and dimer (brown squares), and a low relative abundance charge state distribution for monomer (green circles). With $0.5 \% \mathrm{~m}$ NBA, the average charge state for all three forms of the protein (monomer, dimer, and tetramer) increases significantly, and all three charge state distributions are broader than without $m$-NBA (Table 1; Figure 1b). Modest additional increases in average charge and range of charge states are observed for the tetramer and dimer with $1.0 \%$ and $1.5 \%$ $m$-NBA, whereas the average charge of the monomer 
Table 1. Average Charge (and Range of Charge States) Observed for each Oligomeric State of Concanavalin A Nanoelectrosprayed from $200 \mathrm{mM}$ Ammonium Acetate Solutions Containing 0\%-1.5\% $\mathrm{m}$-NBA

Average charge (and range of charge states) $(+)$

\begin{tabular}{|c|c|c|c|c|}
\hline Concanavalin A & $0 \%$ & $0.5 \%$ & $1.0 \%$ & $1.5 \%$ \\
\hline Tetramer & $20.3(22-19)$ & $23.5(27-21)$ & $24.8(30-22)$ & $25.0(30-22)$ \\
\hline Dimer & $14.3(16-13)$ & $17.4(20-15)$ & $17.8(21-16)$ & $18.0(21-16)$ \\
\hline Monomer & $9.4(10-8)$ & $11.8(15-9)$ & $10.8(16-9)$ & $11.6(17-9)$ \\
\hline
\end{tabular}

decreases somewhat at these higher concentrations (Table 1; Figure 1c-d).

The normalized abundances of the three forms of the protein as a function of $m$-NBA concentration are shown in Figure 1e. There is a significant increase in the normalized abundance of the monomer with $1.0 \%$ and $1.5 \% \mathrm{~m}$-NBA that is concomitant with a decrease in the normalized abundance of the dimer at these higher reagent concentrations. In contrast, the normalized abundance of the tetramer is essentially constant at all reagent concentrations. These results are consistent with $m$-NBA causing dissociation of the dimer into its constituent monomers. All monomer ions have the same or higher $\mathrm{m} / \mathrm{z}$ than the dimer ions. In nozzle-skimmer dissociation experiments that cause gas-phase dissociation of native and supercharged concanavalin A dimers, the product monomer peaks are observed at higher, the same, and lower $\mathrm{m} / \mathrm{z}$ than the average of the precursor dimer distribution owing to predominantly symmetric dissociation [51, 52] (Supplemental Figure 1). The higher charge state monomer ions and the absence of lower charge state monomer ions in the mass spectrum with $m$ NBA (Figure 1b-d) indicate that the dimers dissociate in solution and not in the gas phase where charge is conserved. It is not possible to determine from these data alone whether dissociation occurs prior to ESI or in the ESI droplets.

Previous HDX-MS and CD experiments aimed at elucidating the origin of aqueous solution supercharging show that the native structures of proteins remain intact until the concentration of the supercharging reagent is increased in the ESI droplet owing to preferential evaporation of water [21-23]. To determine if $m$-NBA affects the dimermonomer equilibrium prior to ESI, solutions of the protein were crosslinked with suberic acid bis(3-sulfohydroxysuccinimide ester) with and without $1 \% \mathrm{~m}$-NBA in the crosslinking buffer. Comparison of nanoESI mass spectra obtained for the two crosslinked protein solutions diluted to $90 \%$ formic acid to ensure complete dissociation of noncrosslinked dimers shows that the extent of crosslinking is effectively identical for the two preparations (Supplemental Figure 2). This indicates that $m$-NBA does not measurably affect the dimer-monomer equilibrium prior to ESI. Because the dimer dissociation does not occur in the gas phase, the dimer dissociation observed with $m$-NBA (Figure 1b-d) must occur in the ESI droplet.

The results of TWIMS-MS experiments in which the collision cross sections of concanavalin A tetramer ions are measured as a function of ion charge state are shown in
Figure 2. For the same ion charge states formed from solutions containing different concentrations of $m$-NBA, there are only small differences in the measured collision cross sections, consistent with previous TWIMS-MS arrival time distributions of myoglobin supercharged with $m$-NBA or sulfolane [21]. The collision cross sections of the 19+ to $25+$ charge states are essentially the same, whereas for the $26+$ and higher charge states, the collision cross sections increase significantly with increasing charge state. These results indicate that modest increases in charge as a result of supercharging may occur without gross conformational changes but that beyond some threshold, which is likely protein-specific, enhanced charging may be accompanied by significant changes to the native conformations.

Less supercharging was observed for hen egg white lysozyme when this $14.3 \mathrm{kDa}$ protein was electrosprayed from $200 \mathrm{mM}$ ammonium acetate compared with pure water solutions using dimethyl sulfoxide as the supercharging reagent [22]. Buffers can stabilize folded forms of the protein, and may partially or fully counteract the destabilizing effects of the supercharging reagents during droplet evaporation and formation [22]. To investigate the effects of buffer concentration on the extent of supercharging of concanavalin A, nanoelectrospray mass spectra of $\sim 10 \mu \mathrm{M}$ concanavalin A from solutions containing $1 \% \mathrm{~m}$-NBA and either $600 \mathrm{mM}$ ammonium acetate, $200 \mathrm{mM}$ ammonium acetate, or water (all at $\mathrm{pH} 7.0$ ) were obtained (Figure 3a-c).

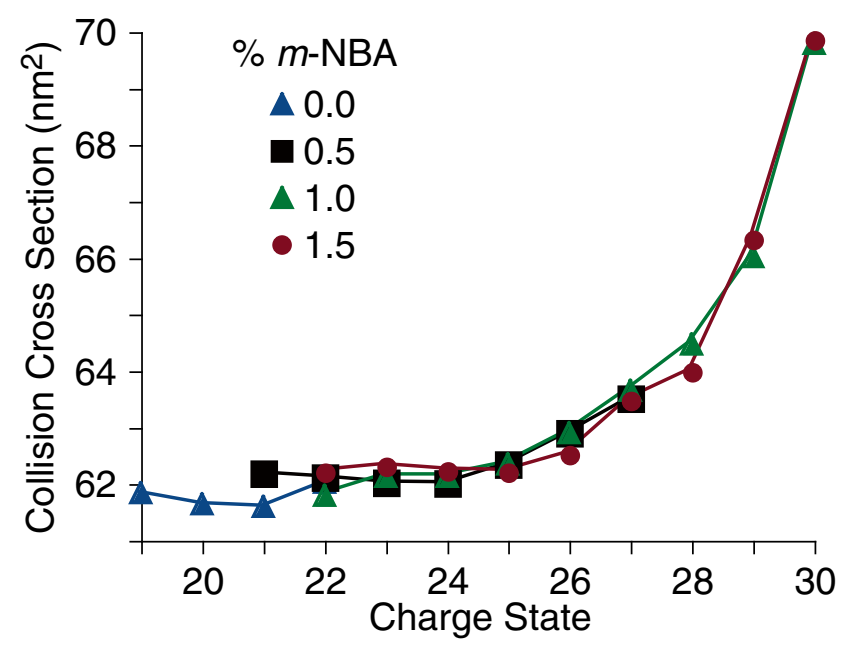

Figure 2. Collision cross section of concanavalin A tetramers as a function of charge state for $\sim 10 \mu \mathrm{M}$ aqueous protein solutions containing $0 \%-1.5 \% \mathrm{~m}$-NBA 

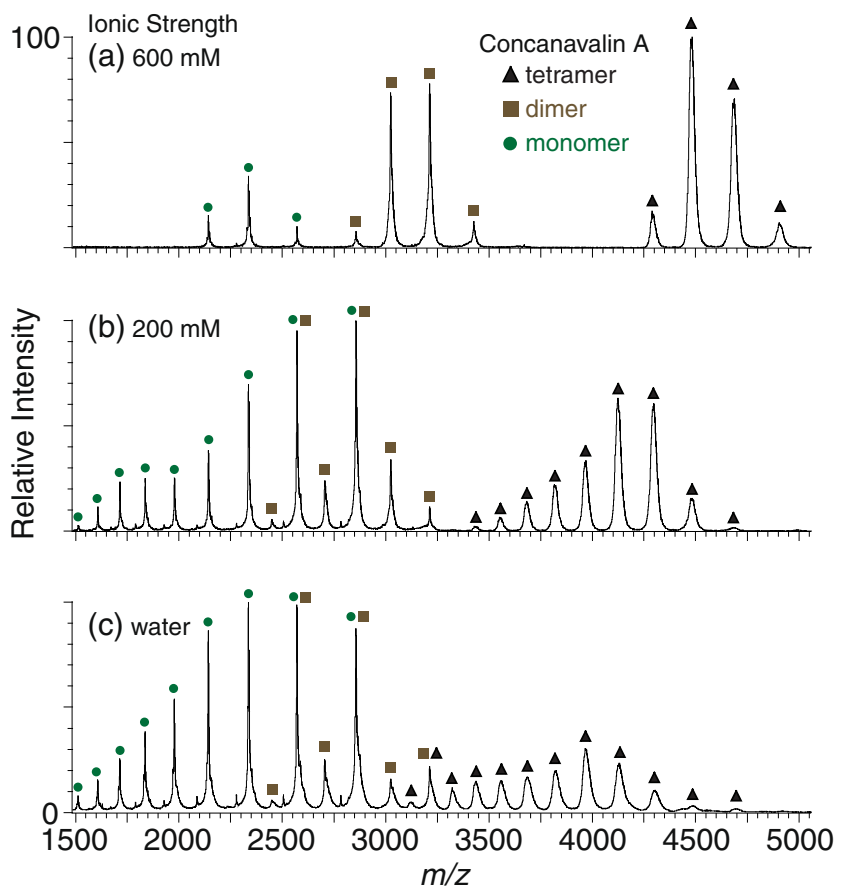

Figure 3. Nanoelectrospray mass spectra of $\sim 10 \mu \mathrm{M}$ aqueous concanavalin $\mathrm{A}$ solutions containing 1\% $\mathrm{m}$-NBA and either (a) $600 \mathrm{mM}$ ammonium acetate, (b) $200 \mathrm{mM}$ ammonium acetate, or (c) water

The average charge and width of the charge state distributions for the tetramer and dimer increase dramatically with decreasing ionic strength. The highest charge state observed for the tetramer is formed from the unbuffered solution (33+; Figure $3 \mathrm{c}$ ) and has a collision cross section of $75.9 \mathrm{~nm}^{2}$, which is $22 \%$ higher than the average collision cross section of the tetramer obtained without $m$-NBA from a $200 \mathrm{mM}$ ammonium acetate solution (Figure 2). The changes in relative abundance of all forms of the protein as a function of $m$-NBA concentration for each of the three ionic strength solutions are shown in Figure 4. There is a significant increase in the normalized abundance of the monomer that is correlated with a decrease in ionic strength and is concomitant with a decrease in the normalized abundance of the dimer. In contrast, the normalized abundance of the tetramer does not change significantly under these solution conditions. The charge state distribution of the monomer ions relative to the charge state distribution of the dimer ions again suggests that dimer dissociation occurs in solution and not in the gas phase. It is not possible to determine from these experiments alone if the increases in charging with decreasing ionic strength are due to dimer and tetramer destabilization, or if other factors such as competition for charge with ammonia in the gas phase or decreased droplet charge due to charge carrier emission [53,54] are also limiting the extent of supercharging. However, these results suggest that the buffer concentration should be minimized when maximum charging with supercharging reagents is desired.

There are a significantly higher number of intermolecular hydrogen bonds ( 52 versus 14 ) and salt bridges (12 versus 0 )
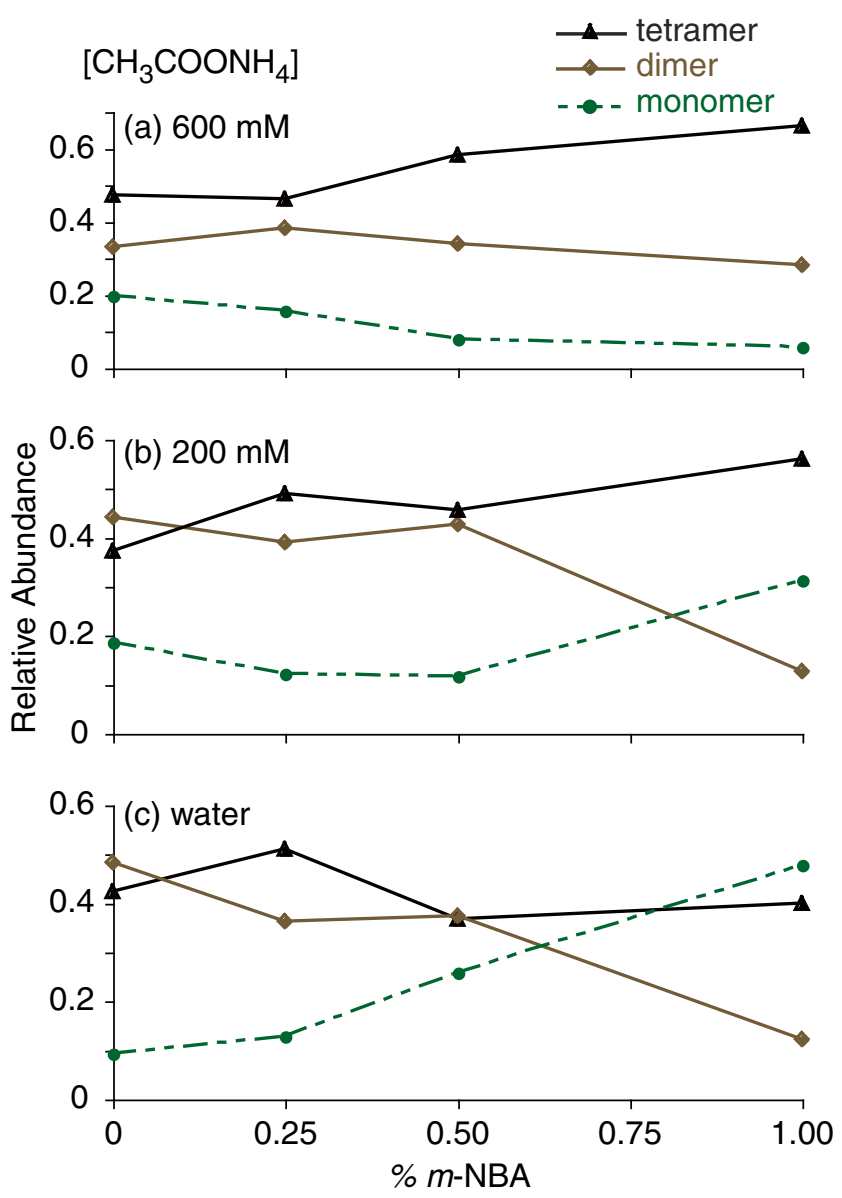

Figure 4. Normalized abundances of concanavalin A monomer, dimer and tetramer as a function of $m$-NBA concentration for $\mathrm{pH} 7.0$ aqueous solutions containing (a) $600 \mathrm{mM}$, (b) $200 \mathrm{mM}$, or (c) no ammonium acetate

for the tetramer compared with the dimer based on the protomer binding information obtained from the crystal structure of concanavalin A [55]. This should lead to a larger activation barrier for dissociation of the tetramer compared with the dimer, consistent with the absence of tetramer dissociation and the extensive dimer dissociation observed in these experiments. Both thermodynamic [48, 49] and crystallographic [55] evidence suggest that the dimer-dimer binding interface of the tetramer is large and hydrophobic. If solvent molecules are sufficiently excluded from this binding interface before and during ESI, the effectiveness of the supercharging reagent as a denaturant may also be reduced [20-23], consistent with the relatively modest extent of supercharging and the absence of tetramer dissociation observed in these experiments.

\section{Complex Destabilization Is Not Always Indicated by a Change in Collision Cross Section}

Bacillus anthracis produces a binary toxin complex that assembles via heptameric [56] and octameric [57] ringshaped homo-oligomers of the protective antigen protein 
(PA). These complexes can form in solution or on host cell surfaces [57]. Proteolytic activation of the $\sim 83 \mathrm{kDa}$ PA results in loss of a $\sim 20 \mathrm{kDa}$ fragment and PA oligomerizes into either a heptameric [58] or octameric prechannel complex [57]. Loss of the $20 \mathrm{kDa}$ fragment exposes binding sites for the two other enzyme components of anthrax toxin, called lethal factor (LF) and edema factor (EF). These heptameric and octameric PA complexes can bind up to three or four copies of LF (or EF), respectively [45, 57]. Following endocytosis, the PA oligomer undergoes an acidic $\mathrm{pH}$-induced conformational transition into its translocase channel state [59]. The enzyme factors, LF and EF, are then trafficked across the cell membrane into the cytosol where they disrupt normal cellular function [60].

Nanoelectrospray mass spectra of $\sim 10 \mu \mathrm{M}$ octamerenriched PA oligomers bound to $\sim 31 \mathrm{kDa}$ PA-binding domain of $L F\left(\mathrm{LF}_{\mathrm{N}}\right)$ [61] from $200 \mathrm{mM}$ ammonium acetate, $\mathrm{pH} 7.8$, solutions containing $0 \%-0.8 \% \mathrm{~m}$-NBA are shown in Figure 5. All ions of the subcomplexes and protein monomers were removed in the quadrupole by allowing only ions with $m / z>8000$ to travel to the IMS and TOF regions of the Synapt G2 instrument [62] used to obtain these data. Without $m$-NBA in the solution, the spectrum is dominated by a single narrow charge state distribution for the octameric complex, with a much lower relative abundance charge state distribution for the heptameric complex (Figure 5a). The abundance-weighted average collision cross sections for the octameric and heptameric complexes are 185 and $163 \mathrm{~nm}^{2}$, respectively, both of which are $95 \%$ of their calculated collision cross section using the projection approximation algorithm in MOBCAL [44] with the crystal structure coordinates of the octameric complex [45] and a homology model of the heptameric complex. The good agreement between the measured and calculated collision cross section suggests that these complexes do not undergo gross conformational changes during ESI or in transit to the ion mobility cell [63]. With $0.4 \% \mathrm{~m}$-NBA in the electrospray solution, the average charge for the octameric complex increases modestly from $49.0+$ to $50.5+$ and the average collision cross section also increases slightly from 185 to $187 \mathrm{~nm}^{2}$. There is a significant decrease in ion abundance for the heptameric complex so that it is not possible to measure the average charge or collision cross section. A small population of high-charge $\mathrm{LF}_{\mathrm{N}}$ monomers is observed in the range $\mathrm{m} / \mathrm{z} 1200$ 1800 . With $0.8 \% \mathrm{~m}$-NBA in the electrospray solution, there is a dramatic decrease in abundance for the octameric complex and a significant increase in the abundance of the high-charge $\mathrm{LF}_{\mathrm{N}}$ ions (Figure $5 \mathrm{c}$ ). There is also a low relative abundance charge state distribution corresponding to a $\mathrm{PA}_{2} \mathrm{LF}_{\mathrm{N}}$ subcomplex in the range $m / z$ 3600-4600. These lower mass $\mathrm{LF}_{\mathrm{N}}$ monomer and subcomplex ions must be the products of gas-phase dissociation in a region of the instrument beyond the massfiltering quadrupole $(\mathrm{m} / \mathrm{z}>8000)$. The average charge of the small population of octameric complexes that survives intact

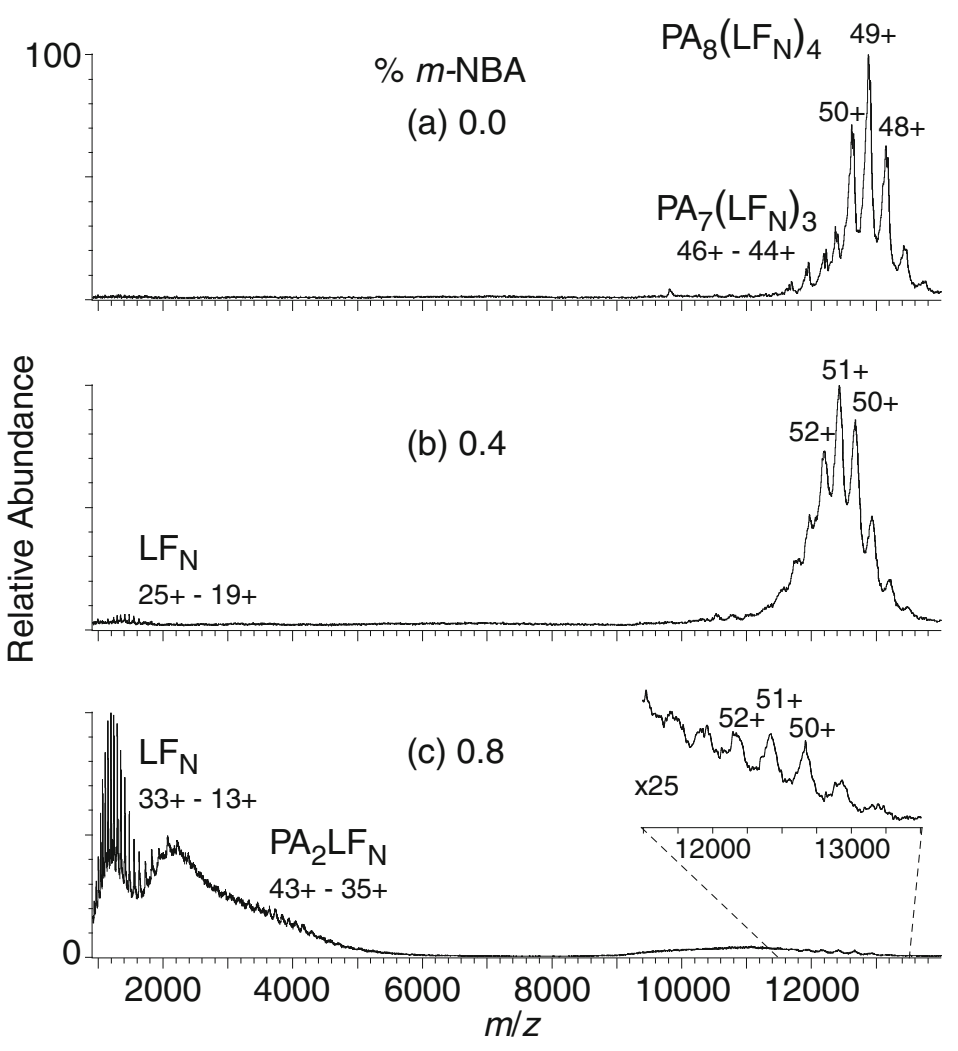

Figure 5. Nanoelectrospray mass spectra of $\sim 10 \mu \mathrm{M}$ octamer-enriched PA oligomers bound to $\mathrm{LF}_{\mathrm{N}}$ from $200 \mathrm{mM}$ ammonium acetate, $\mathrm{pH} 7.8$, aqueous solutions containing (a) $0 \%$, (b) $0.4 \%$, and (c) $0.8 \% \mathrm{~m}$-NBA 
(Figure 5c inset) is $50.8+$ and the average collision cross section is $185 \mathrm{~nm}^{2}$.

In contrast to the solution-phase dissociation caused by $m$-NBA for supercharged concanavalin A dimers, a significant population of the supercharged octameric complex survives intact long enough to pass the mass filtering quadrupole but then dissociates in the gas phase, most likely in the relatively high-pressure regions of the ion mobility section of the instrument as a result of unintentional collisional activation. The small population of octameric complexes that survives intact has essentially the same average charge as those from the $0.4 \% \mathrm{~m}$-NBA solution $(50.8+$ versus $50.5+)$ and a slightly lower average collision cross section $\left(185 \mathrm{~nm}^{2}\right.$ versus $\left.187 \mathrm{~nm}^{2}\right)$. These results indicate that only very small changes in the rotationallyaveraged size of the complex ion occur as a result of the $m$ NBA enrichment, whereas the extensive gas-phase dissociation of the intact octamer indicates that the native structure and subunit interactions are significantly destabilized. This destabilization could occur first in the ESI droplet as a result of $m$-NBA-induced chemical and/or thermal denaturation [19-23], followed by either metastable or collisionally-activated dissociation (CAD) after the mass-filtering quadrupole.

To determine if destabilization occurs instead as result of increased Coulombic repulsion after the higher charge state ions are formed, the average charge of the octamer complex was increased by inducing a specific conformational change in the complex that occurs away from the PA-ligand binding interface [42], where the vast majority of dissociation occurs for the supercharged complex. TWIMS-MS spectra of these complexes were acquired with the same instrument conditions but without $m$-NBA. The conformational transition of anthrax toxin complexes from the cell-surface prechannel state to the transmembrane channel state was revealed in previous MS experiments by a subtle increase in the average and maximum charge in the $\mathrm{pH}$ range 7.2-7.0 that occurs without a shift in the charge states of the subcomplexes or constituent monomers, indicating a specific conformational change of the complex rather than just $\mathrm{pH}$-induced unfolding [42]. The transition midpoint observed using electron microscopy (EM) and $\mathrm{CD}$ was also in the $\mathrm{pH}$ range $7.2-$ 7.0 [42]. Here, the $\mathrm{pH}$ titration was performed with the same octamer-enriched sample of anthrax toxin and with the same instrument conditions as was used in the supercharging experiments, but without $m$-NBA. Nanoelectrospray mass spectra obtained from the $\mathrm{pH} 7.2$ and 7.0 solutions (Figure 6) show a subtle increase in the average charge at the lower $\mathrm{pH}$ without any significant changes to the complex abundance or additional gas-phase fragmentation. Mass spectra from the $\mathrm{pH}$ 7.8-7.2 solutions are all essentially identical, and mass spectra from the $\mathrm{pH} 7.0-6.6$ solutions are also all essentially identical (Supplemental Figure 3). The low abundance $\mathrm{LF}_{\mathrm{N}}$ monomers are present in all spectra from $\mathrm{pH}$ 7.6-6.6 with approximately equal signal/noise. A plot of the average charge and average collision cross section as a function of

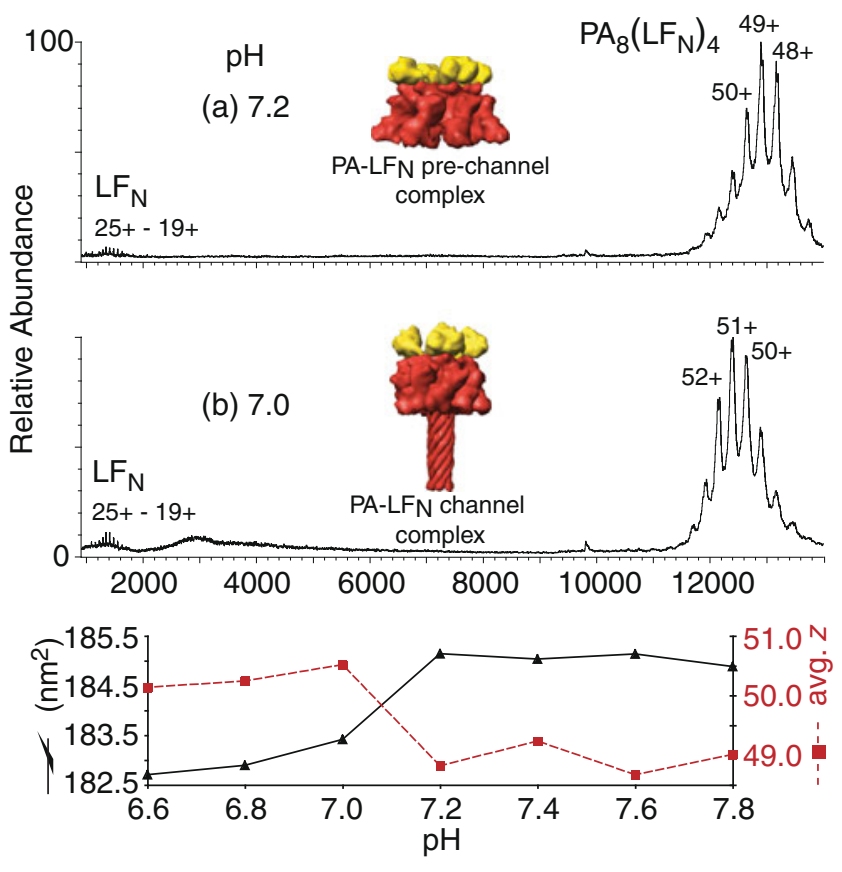

Figure 6. Nanoelectrospray mass spectra of $\sim 10 \mu \mathrm{M}$ octamer-enriched PA oligomers bound to $L_{\mathrm{N}}$ from $200 \mathrm{mM}$ ammonium acetate aqueous solutions at $\mathrm{pH}$ (a) 7.2 and (b) 7.0. Models of the prechannel and channel forms of anthrax toxin complexes are inset. Plotted below the mass spectra are the average charge (red squares; right axis) and average collision cross section (black triangles; left axis) of the octamer complex as a function of solution $\mathrm{pH}$

$\mathrm{pH}$ is shown in Figure 6c, which demonstrates that the shift to modestly higher charge states between $\mathrm{pH} 7.2$ and 7.0 is concomitant with an overall compaction of the complex as measured by a subtle decrease in the collision cross section. This is an interesting result because higher charge states for proteins are normally associated with more unfolded or elongated structures, but in this case, the rotationally-averaged size becomes more compact and results in higher charging. The oligomer in the prevailing model for the structure of the PA channel forms an approximately $100-\AA \AA$ long $\beta$-barrel domain $[42,64]$, and this extended domain appears to be able to accommodate more charges (Figure 6 inset).

The gas-phase structures of the channel state of the toxin complex are somewhat more compact than the supercharged prechannel state complex $\left(183 \mathrm{~nm}^{2}\right.$ versus $185 \mathrm{~nm}^{2}$, respectively), with marginally lower average charge $(50.5+$ versus $50.8+$, respectively). The ratio of collision cross section/charge is the same for these two forms of the complex $\left(3.6 \mathrm{~nm}^{2} / z\right)$, indicating that the Coulombic repulsion should be essentially the same for the channel state complex and the supercharged prechannel complex. However, the extensive dissociation observed with supercharging of the prechannel complex is not observed in any of the channel complex spectra (Supplemental Figure 3). This demonstrates that dissociation of $\mathrm{LF}_{\mathrm{N}}$ from the supercharged prechannel toxin 
complex does not occur as a result of increased Coulombic repulsion, but rather as a result of destabilization that occurs prior to ion formation, similar to the destabilization that occurs for concanavalin A dimers.

\section{Chemical and/or Thermal Destabilization in the ESI Droplet}

All of the results of our studies aimed at elucidating the origin of aqueous solution supercharging have supported the hypothesis that the broad distribution of high charge ions observed with "native" protein and complex supercharging are caused by chemical and/or thermal denaturation of the analyte in the ESI droplet as the reagent concentration increases due to preferential evaporation of the solvent [1923]. The TWIMS-MS results presented here indicate that chemical and/or thermal "destabilization" can occur without an extensive unfolding process. This destabilization must be sufficient for accommodation of additional charges, but may not necessarily result in significant changes to the rotationally averaged size of the ion after its formation.

The prechannel to channel transition for anthrax toxin complexes occurs with a significant change in their solutionphase structures [42, 64-66]. Nonetheless, only a modest $\sim 1 \%$ decrease in the collision cross section (Figure $6 \mathrm{c}$ ) was measured for the octamer channel compared with the octamer prechannel. This illustrates that significant changes in solution-phase structures do not always result in significant changes in the collision cross section. Similarly, supercharged prechannel complexes have essentially unchanged collision cross sections compared with native prechannel complexes, but the extensive gas-phase dissociation of the former clearly indicates that $m$-NBA causes destabilization of its native structure that is not related to an increase in columbic repulsion after ion formation. Only the highest charge concanavalin A tetramers had measurably different collision cross sections (Figure 2), yet the dramatic dissociation of the dimers in the ESI droplet (Figure 3) clearly indicates that $m$-NBA is affecting the native dimermonomer equilibrium.

Native solution supercharging of proteins and protein complexes has been used in a variety of studies, including comparisons of collision cross sections, CAD fragmentation pathways, and dissociation energies as a function of charge state [30]; attempts to map the binding sites of a cofactor [31] or ligands [32] utilizing top-down dissociation techniques; and for small molecule screening [33]. In each of these studies, the structures of the analytes formed by supercharging were assumed to be the same as those originating from buffered solutions without the supercharging reagent. The results described here show that this may not always be the case, and that the potential for destabilization or more significant denaturation and unfolding of the analyte [19-23] should be considered when supercharging reagents are used.

\section{Conclusions}

The results of these TWIMS-MS studies of supercharged concanavalin A oligomers and anthrax toxin octamer indicate that charge enhancement is the result of destabilization of the native solution-phase structures that may or may not be reflected as changes in the collision cross sections of the gas-phase ions. Similar to what occurs with monomeric proteins, this destabilization appears to happen in the ESI droplet as water preferentially evaporates and the droplet becomes enriched in the high-boiling point reagent, which then acts as a chemical denaturant [19-23]. Concanavalin A oligomers are supercharged to a much greater extent in water versus 200 and $600 \mathrm{mM}$ ammonium acetate solutions, concomitant with much more extensive solutionphase dissociation of the dimer and denaturation of the tetramer. Further elucidation of the role of salts in charging [67] and supercharging is required, but these results indicate that buffer or other salt concentrations should be minimized when maximum charge through the use of supercharging reagents is desired.

Despite the destabilization inherent to aqueous solution supercharging, this technique has potential for the development or improvement of ESI-MS applications that provide information about the structures, structural transitions, and dynamics of biomolecules, as well as their complexes in solution [23, 32] and in the gas phase [31]. In applications in which rapid unfolding and/or subunit dissociation is desired but supercharging reagents are not sufficiently denaturing on the timescale of ESI, it may be possible to use low concentrations of some Hofmeister salts to partially destabilize the equilibrium conformations prior to, and during, ESI and/or to introduce acid vapors in the countercurrent drying gas to lower the droplet $\mathrm{pH}$ during ESI, as demonstrated by McLuckey and coworkers [68]. This latter technique also works best in unbuffered solutions [68], so it should be particularly effective when combined with native supercharging from unbuffered solution.

\section{Acknowledgments}

The authors thank the National Institutes of Health (training grants T32GM008295 for H.J.S. and T32GM066698 for A. F.K., and grants R01-AI077703 for B.A.K, and R01GM096097 for E.R.W.) for generous financial support. The authors thank Professor Ewa Witkowska and Dr. Haichuan Liu at the UCSF Sandler-Moore Mass Spectrometry Core Facility for use of the Synapt G2 instrument (N.I. H. grant 1S10RR029446-01).

\section{References}

1. Dobo, A., Kaltashov, I.A.: Detection of Multiple Protein Conformational Ensembles in Solution Via Deconvolution of Charge-State Distributions in ESI MS. Anal. Chem. 73, 4763-4773 (2001)

2. Chowdhury, S.K., Katta, V., Chait, B.T.: Probing Conformational Changes in Proteins by Mass Spectrometry. J. Am. Chem. Soc. 112, 9012-9013 (1990) 
3. Loo, J.A., Loo, R.R.O., Udseth, H.R., Edmonds, C.G., Smith, R.D.: Solvent-Induced Conformational Changes of Polypeptides Probed by Electrospray-Ionization Mass-Spectrometry. Rapid Commun. Mass Spectrom. 5, 101-105 (1991)

4. Iavarone, A.T., Jurchen, J.C., Williams, E.R.: Effects of Solvent on the Maximum Charge State and Charge State Distribution of Protein Ions Produced by Electrospray Ionization. J. Am. Soc. Mass Spectrom. 11, 976-985 (2000)

5. Loo, R.R.O., Smith, R.D.: Proton-Transfer Reactions of MultiplyCharged Peptide and Protein Cations and Anions. J. Mass Spectrom. 30, 339-347 (1995)

6. Williams, E.R.: Proton Transfer Reactivity of Large Multiply Charged Ions. J. Mass Spectrom. 31, 831-842 (1996)

7. Iavarone, A.T., Jurchen, J.C., Williams, E.R.: Supercharged Protein and Peptide Ions Formed by Electrospray Ionization. Anal. Chem. 73, 1455$1460(2001)$

8. Iavarone, A.T., Williams, E.R.: Supercharging in Electrospray Ionization: Effects on Signal and Charge. Int. J. Mass Spectrom. 219, 63-72 (2002)

9. Iavarone, A.T., Williams, E.R.: Mechanism of Charging and Supercharging Molecules in Electrospray Ionization. J. Am. Chem. Soc. 125, 2319-2327 (2003)

10. Benkestock, K., Sundqvist, G., Edlund, P.O., Roeraade, J.: Influence of Droplet Size, Capillary-Cone Distance and Selected Instrumental Parameters for the Analysis of Noncovalent Protein-Ligand Complexes by Nano-Electrospray Ionization Mass Spectrometry. J. Mass Spectrom. 39, 1059-1067 (2004)

11. Page, J.S., Kelly, R.T., Tang, K., Smith, R.D.: Ionization and Transmission Efficiency in an Electrospray Ionization-Mass Spectrometry Interface. J. Am. Soc. Mass Spectrom. 18, 1582-1590 (2007)

12. Thomson, B.A.: Declustering and Fragmentation of Protein Ions from an Electrospray Ion Source. J. Am. Soc. Mass Spectrom. 8, 1053-1058 (1997)

13. Yang, P.X., Cooks, R.G., Ouyang, Z., Hawkridge, A.M., Muddiman, D. C.: Gentle Protein Ionization Assisted by High-Velocity Gas Flow. Anal. Chem. 77, 6174-6183 (2005)

14. Scalf, M., Westphall, M.S., Smith, L.M.: Charge Reduction Electrospray Mass Spectrometry. Anal. Chem. 72, 52-60 (2000)

15. Stephenson, J.L., McLuckey, S.A.: Charge Manipulation for Improved Mass Determination of High-Mass Species and Mixture Components by Electrospray Mass Spectrometry. J. Mass Spectrom. 33, 664-672 (1998)

16. Catalina, M.I., van den Heuvel, R.H.H., van Duijn, E., Heck, A.J.R.: Decharging of Globular Proteins and Protein Complexes in Electrospray. Chem. Eur. J. 11, 960-968 (2005)

17. Iavarone, A.T., Williams, E.R.: Collisionally Activated Dissociation of Supercharged Proteins Formed by Electrospray Ionization. Anal. Chem. 75, 4525-4533 (2003)

18. Lomeli, S.H., Yin, S., Loo, R.R.O., Loo, J.A.: Increasing Charge While Preserving Noncovalent Protein Complexes for ESI-MS. J. Am. Soc. Mass Spectrom. 20, 593-596 (2009)

19. Sterling, H.J., Williams, E.R.: Origin of Supercharging in Electrospray Ionization of Noncovalent Complexes from Aqueous Solution. J. Am. Soc. Mass Spectrom. 20, 1933-1943 (2009)

20. Sterling, H.J., Cassou, C.A., Trnka, M.J., Burlingame, A.L., Krantz, B. A., Williams, E.R.: The Role of Conformational Flexibility on Protein Supercharging in Native Electrospray Ionization. Phys. Chem. Chem. Phys. 13, 18288-18296 (2011)

21. Sterling, H.J., Daly, M.P., Feld, G.K., Thoren, K.L., Kintzer, A.F., Krantz, B.A., Williams, E.R.: Effects of Supercharging Reagents on Noncovalent Complex Structure in Electrospray Ionization from Aqueous Solutions. J. Am. Soc. Mass Spectrom. 21, 1762-1774 (2010)

22. Sterling, H.J., Prell, J.S., Cassou, C.A., Williams, E.R.: Protein Conformation and Supercharing with DMSO from Aqueous Solution. J. Am. Soc. Mass Spectrom. 22, 1178-1186 (2011)

23. Sterling, H.J., Williams, E.R.: Real-Time Hydrogen/Deuterium Exchange Kinetics Via Supercharged Electrospray Ionization Tandem Mass Spectrometry. Anal. Chem. 82, 9050-9057 (2010)

24. Lomeli, S.H., Peng, I.X., Yin, S., Loo, R.R.O., Loo, J.A.: New Reagents for Increasing ESI Multiple Charging of Proteins and Protein Complexes. J. Am. Soc. Mass Spectrom. 21, 127-131 (2010)

25. Davies, N.W., Wiese, M.D., Browne, S.G.A.: Characterization of Major Peptides in 'Jack Jumper' Ant Venom by Mass Spectrometry. Toxicon 43, 173-183 (2004)
26. Kjeldsen, F., Giessing, A.M.B., Ingrell, C.R., Jensen, O.N.: Peptide Sequencing and Characterization of Post-Translational Modifications by Enhanced Ion-Charging and Liquid Chromatography Electron-Transfer Dissociation Tandem Mass Spectrometry. Anal. Chem. 79, 9243-9252 (2007)

27. Madsen, J.A., Brodbelt, J.S.: Comparison of Infrared Multiphoton Dissociation and Collision-Induced Dissociation of Supercharged Peptides in Ion Traps. J. Am. Soc. Mass Spectrom. 20, 349-358 (2009)

28. Sze, S.K., Ge, Y., Oh, H., McLafferty, F.W.: Top-Down Mass Spectrometry of a 29-kDa Protein for Characterization of Any Posttranslational Modification to within One Residue. Proc. Natl. Acad. Sci. U.S.A. 99, 1774-1779 (2002)

29. Valeja, S.G., Tipton, J.D., Emmett, M.R., Marshall, A.G.: New Reagents for Enhanced Liquid Chromatographic Separation and Charging of Intact Protein Ions for Electrospray Ionization Mass Spectrometry. Anal. Chem. 82, 7515-7519 (2010)

30. Erba, E.B., Ruotolo, B.T., Barsky, D., Robinson, C.V.: Ion MobilityMass Spectrometry Reveals the Influence of Subunit Packing and Charge on the Dissociation of Multiprotein Complexes. Anal. Chem. 82, 9702-9710 (2010)

31. Enyenihi, A.A., Yang, H., Ytterberg, A.J., Lyutvinskiy, Y., Zubarev, R.A.: Heme Binding in Gas-Phase Holo-Myoglobin Cations: Distal Becomes Proximal? J. Am. Soc. Mass Spectrom. 22, 1763-1770 (2011)

32. Yin, S., Loo, J.A.: Top-Down Mass Spectrometry of Supercharged Native Protein-Ligand Complexes. Int. J. Mass Spectrom. 300, 118-122 (2011)

33. Regazzoni, L., Bertoletti, L., Vistoli, G., Colombo, R., Aldini, G., Serra, M., Carini, M., Caccialanza, G., De Lorenzi, E.: A Combined HighResolution Mass Spectrometric and in Silico Approach for the Characterization of Small Ligands of $\beta(2)$-Microglobulin. Chem. Med. Chem. 5, 1015-1025 (2010)

34. Hogan, C.J., Loo, R.R.O., Loo, J.A., de la Mora, J.F.: Ion MobilityMass Spectrometry of Phosphorylase B Ions Generated with Supercharging Reagents but in Charge-Reducing Buffer. Phys. Chem. Chem. Phys. 12, 13476-13483 (2010)

35. Grimm, R.L., Beauchamp, J.L.: Evaporation and Discharge Dynamics of Highly Charged Multicomponent Droplets Generated by Electrospray Ionization. J. Phys. Chem. A 114, 1411-1419 (2010)

36. Ahadi, E., Konermann, L.: Ejection of Solvated Ions from Electrosprayed Methanol/Water Nanodroplets Studied by Molecular Dynamics Simulations. J. Am. Chem. Soc. 133, 9354-9363 (2011)

37. Zhou, S.L., Cook, K.D.: Probing Solvent Fractionation in Electrospray Droplets with Laser-Induced Fluorescence of a Solvatochromic Dye. Anal. Chem. 72, 963-969 (2000)

38. CRC Handbook of Chemistry and Physics. In [Online] 89th ed.; D. R. Lide, Ed. 2008-2009.

39. Goodman, J.M., Kirby, P.D., Haustedt, L.O.: Some Calculations for Organic Chemists: Boiling Point Variation, Boltzmann Factors and the Eyring Equation. Tetrahedron Lett. 41, 9879-9882 (2000)

40. Lord Rayleigh Phils. Mag. 1882, 14, 184-186.

41. Scherrenberg, R., Coussens, B., van Vliet, P., Edouard, G., Brackman, J., de Brabander, E., Mortensen, K.: The Molecular Characteristics of Poly(Propyleneimine) Dendrimers as Studied with Small-Angle Neutron Scattering, Viscosimetry, and Molecular Dynamics. Macromolecules 31, 456-461 (1998)

42. Kintzer, A.F., Sterling, H.J., Tang, I.I., Abdul-Gader, A., Miles, A.J., Wallace, B.A., Williams, E.R., Krantz, B.A.: Role of the Protective Antigen Octamer in the Molecular Mechanism of Anthrax Lethal Toxin Stabilization in Plasma. J. Mol. Biol. 399, 741-758 (2010)

43. Bush, M.F., Hall, Z., Giles, K., Hoyes, J., Robinson, C.V., Ruotolo, B. T.: Collision Cross Sections of Proteins and Their Complexes: A Calibration Framework and Database for Gas-Phase Structural Biology. Anal. Chem. 82, 9557-9565 (2010)

44. Mesleh, M.F., Hunter, J.M., Shvartsburg, A.A., Schatz, G.C., Jarrold, M.F.: Structural Information from Ion Mobility Measurements: Effects of the Long-Range Potential. J. Phys. Chem. 100, 16082-16086 (1996)

45. Feld, G.K., Thoren, K.L., Kintzer, A.F., Sterling, H.J., Tang, I.I., Greenberg, S.G., Williams, E.R., Krantz, B.A.: Structural Basis for the Unfolding of Anthrax Lethal Factor by Protective Antigen Oligomers. Nat. Struct. Mol. Biol. 17, 1383-1390 (2010)

46. Emsley, P., Cowtan, K.: Coot: Model-Building Tools for Molecular Graphics. Acta Crystallogr. D: Biol. Crystallogr. 60, 2126-2132 (2004) 
47. Lacy, D.B., Wigelsworth, D.J., Melnyk, R.A., Harrison, S.C., Collier, R.J.: Structure of Heptameric Protective Antigen Bound to an Anthrax Toxin Receptor: A Role for Receptor in $\mathrm{pH}-$ Dependent Pore Formation. Proc. Natl. Acad. Sci. U.S.A. 101, 13147-13151 (2004)

48. Huet, M., Claverie, J.M.: Sedimentation Studies of Reversible DimerTetramer Transition Kinetics of Concanavalin-A. Biochemistry 17, 236$241(1978)$

49. Senear, D.F., Teller, D.C.: Thermodynamics of Concanavalin a DimerTetramer Self-Association: Sedimentation Equilibrium Studies. Biochemistry 20, 3076-3083 (1981)

50. Herskovits, T.T., Jacobs, R., Nag, K.: The Effects of Salts and Ureas on the Subunit Dissociation of Concanavalin-A. Biochim. Biophys. Acta 742, 142-154 (1983)

51. Jurchen, J.C., Garcia, D.E., Williams, E.R.: Further Studies on the Origins of Asymmetric Charge Partitioning in Protein Homodimers. $J$. Am. Soc. Mass Spectrom. 15, 1408-1415 (2004)

52. Jurchen, J.C., Williams, E.R.: Origin of Asymmetric Charge Partitioning in the Dissociation of Gas-Phase Protein Homodimers. J. Am. Chem. Soc. 125, 2817-2826 (2003)

53. Hogan Jr., C.J., Carroll, J.A., Rohrs, H.W., Biswas, P., Gross, M.L. Charge Carrier Field Emission Determines the Number of Charges on Native State Proteins in Electrospray Ionization. J. Am. Chem. Soc. 130, 6926-6927 (2008)

54. Hogan, C.J., Carroll, J.A., Rohrs, H.W., Biswas, P., Gross, M.L.: Combined Charged Residue-Field Emission Model of Macromolecular Electrospray Ionization. Anal. Chem. 81, 369-377 (2009)

55. Reeke, G.N., Becker, J.W., Edelman, G.M.: Covalent and ThreeDimensional Structure of Concanavalin A IV. Atomic Coordinates, Hydrogen Bonding, and Quaternary Structure. J. Biol. Chem. 250, 1525-1547 (1975)

56. Milne, J.C., Furlong, D., Hanna, P.C., Wall, J.S., Collier, R.J.: Anthrax Protective Antigen Forms Oligomers During Intoxication of Mammalian Cells. J. Biol. Chem. 269, 20607-20612 (1994)

57. Kintzer, A.F., Thoren, K.L., Sterling, H.J., Dong, K.C., Feld, G.K., Tang, I.I., Zhang, T.T., Williams, E.R., Berger, J.M., Krantz, B.A.: The Protective Antigen Component of Anthrax Toxin Forms Functional Octameric Complexes. J. Mol. Biol. 392, 614-629 (2009)
58. Petosa, C., Collier, R.J., Klimpel, K.R., Leppla, S.H., Liddington, R.C. Crystal Structure of the Anthrax Toxin Protective Antigen. Nature 385, 833-838 (1997)

59. Blaustein, R.O., Koehler, T.M., Collier, R.J., Finkelstein, A.: Anthrax Toxin-Channel-Forming Activity of Protective Antigen in Planar Phospholipid-Bilayers Proc. Natl. Acad. Sci. U.S.A. 86, 2209-2213 (1989)

60. Krantz, B.A., Finkelstein, A., Collier, R.J.: Protein Translocation through the Anthrax Toxin Transmembrane Pore Is Driven by a Proton Gradient. J. Mol. Biol. 355, 968-979 (2006)

61. Lacy, D.B., Mourez, M., Fouassier, A., Collier, R.J.: Mapping the Anthrax Protective Antigen Binding Site on the Lethal and Edema Factors. J. Biol. Chem. 277, 3006-3010 (2002)

62. Giles, K., Williams, J.P., Campuzano, I.: Enhancements in Traveling Wave Ion Mobility Resolution. Rapid Commun. Mass Spectrom. 25, 1559-1566 (2011)

63. Ruotolo, B.T., Giles, K., Campuzano, I., Sandercock, A.M., Bateman, R.H., Robinson, C.V.: Evidence for Macromolecular Protein Rings in the Absence of Bulk Water. Science 310, 1658-1661 (2005)

64. Katayama, H., Wang, J., Tama, F., Chollet, L., Gogol, E.P., Collier, R. J., Fisher, M.T.: Three-Dimensional Structure of the Anthrax Toxin Pore Inserted into Lipid Nanodiscs and Lipid Vesicles. Proc. Natl. Acad. Sci. U.S.A. 107, 3453-3457 (2010)

65. Vernier, G., Wang, J., Jennings, L.D., Sun, J., Fischer, A., Song, L.K., Collier, R.J.: Solubilization and Characterization of the Anthrax Toxin Pore in Detergent Micelles. Prot. Sci. 18, 1882-1895 (2009)

66. Katayama, H., Janowiak, B.E., Brzozowski, M., Juryck, J., Falke, S., Gogol, E.P., Collier, R.J., Fisher, M.T.: GroEL as a Molecular Scaffold for Structural Analysis of the Anthrax Toxin Pore. Nat. Struct. Mol. Biol. 15, 754-760 (2008)

67. Wang, G.D., Cole, R.B.: Effect of Solution Ionic-Strength on Analyte Charge-State Distributions in Positive and Negative-Ion Electrospray Mass-Spectrometry. Anal. Chem. 66, 3702-3708 (1994)

68. Kharlamova, A., Prentice, B.M., Huang, T.Y., McLuckey, S.A.: Electrospray Droplet Exposure to Gaseous Acids for the Manipulation of Protein Charge State Distributions. Anal. Chem. 82, 7422-7429 (2010) 